\title{
Clarificação de conceitos fundamentais em Cinética Química
}

\section{Introdução}

No ensino da Cinética Química são utilizados correntemente algumas simplificaçð̃es, as quais, se não forem acompanhadas do registo dos respectivos limites de aplicabilidade, podem conduzir a erros graves. É o caso, por exemplo, das leis de velocidade de reacçð̃es químicas de ordem bem definida ou da definição de passo determinante da velocidade reaccional (1-3).

Por outro lado, no cálculo de funçð̌es termodinâmicas de activação, quer se trate do parâmetro de Arrhenius, $\mathrm{E}_{\mathrm{a}}$, obtido pela expressão empírica:

1n $\mathrm{k}=\ln \mathrm{A}-\mathrm{E}_{\mathrm{a}} / \mathrm{RT}$

onde A representa o número de colisão, quer da entalpia, entropia, capacidade calorífica, volume, etc., por aplicação do formalismo termodinâmico à equação fundamental da Teoria do Estado de Transição,

$\ln \mathrm{k}=\ln \left(\frac{\mathrm{RT}}{\mathrm{N}_{\mathrm{A}}} \mathrm{K} \neq / \mathrm{h}\right)$

sendo $\mathrm{RT} / \mathrm{N}_{\mathrm{A}} \mathrm{h}$ o factor de frequência universal e $\mathrm{K} \neq$ uma pseudo-constante de equilíbrio, têm-se detectado fontes de erro que residem na utilização de valores de constantes de velocidade expressas em diferentes unidades $(1,4,5)$.

\section{Desenvolvimento}

\subsection{Ordem de reacção}

É comum considerar equivalentes as expressð̃es «reacção química de ordem $\mathrm{n}$ em relação ao reagente $\mathrm{A}$ » $\mathrm{e}$ " $-\mathrm{dC}_{\mathrm{A}} / \mathrm{dt}=\mathrm{k} \mathrm{C}_{\mathrm{R}^{\prime}}^{\mathrm{n}}$.

No entanto, se se escrever a equação de balanço energético aplicável a $\mathrm{A}$, num elemento de volume $\mathrm{dV}$, num tempo dt:

$\mathrm{dn}_{\mathrm{A}}=\mathrm{dn}_{\mathrm{A}}(\mathrm{I})-\mathrm{dn}_{\mathrm{A}}(\mathrm{II})+\mathrm{dn}_{\mathrm{A}}(\mathrm{III})-\mathrm{dn}_{\mathrm{A}}(\mathrm{IV})$

na qual,

$\mathrm{dn}_{\mathrm{A}}$ - aumento do $\mathrm{n} .^{\circ}$ de moles de $\mathrm{A}$ em $\mathrm{dV}$, durante $\mathrm{dt}$

$\mathrm{dn}_{\mathrm{A}}$ (I) $-\mathrm{n} .^{\circ}$ de moles de $\mathrm{A}$ que entram em $\mathrm{dV}$, durante $\mathrm{dt}$

$\mathrm{dn}_{\mathrm{A}}$ (II) $-\mathrm{n} .^{\circ}$ de moles de $\mathrm{A}$ que deixam $\mathrm{dV}$, durante dt

$\mathrm{dn}_{\mathrm{A}}$ (III) $-\mathrm{n} .^{\circ}$ de moles de A produzidas em $\mathrm{dV}$, durante dt

$\mathrm{dn}_{\mathrm{A}}$ (IV) $-\mathrm{n} .^{\circ}$ de moles de A destruídas em $\mathrm{dV}$, durante dt,

sendo os processos (I) e (II) de transporte de massa e (III) e (IV) reaccionais, é evidente que a lei cinética se deverá referir exclusivamente ao último termo, isto é,
$-\mathrm{dn}_{\mathrm{A}}(\mathrm{IV}) / \mathrm{dt}=\mathrm{k} \mathrm{C}_{\mathrm{A}}^{\mathrm{n}} \mathrm{dV}$

Esta equação apenas se reduzirá à inicial se forem cumpridas certas condiçðes, tal como se indica no esquema 1.

Pode concluir-se, então, que a lei de velocidade, $-\mathrm{dC}_{\mathrm{A}} / \mathrm{dt}=\mathrm{k} \mathrm{C}_{\mathrm{A}}^{\mathrm{n}}$ só é aplicável a uma reacção de ordem n se esta for cineticamente irreversível, conduzida a volume constante e em condiçðes de temperatura e pressão uniformes.

A maioria dos estudos cinéticos de investigação e de ensino são efectuados em vasos reaccionais a temperaratura constante. Nas reacçðes em fase gasosa é usual manter-se constante o volume e em fase líquida a pressão, embora, neste último caso, variaçðes de volume se possam considerar, em geral, desprezáveis.

As condiçð̄es requeridas ao uso daquela expressão são, pois, na prática, aproximadamente cumpridas, o que não invalida o facto de ser necessário, pelo menos em termos teóricos, chamar a atenção para os limites de aplicabilidade das vulgarizadas leis cinéticas.

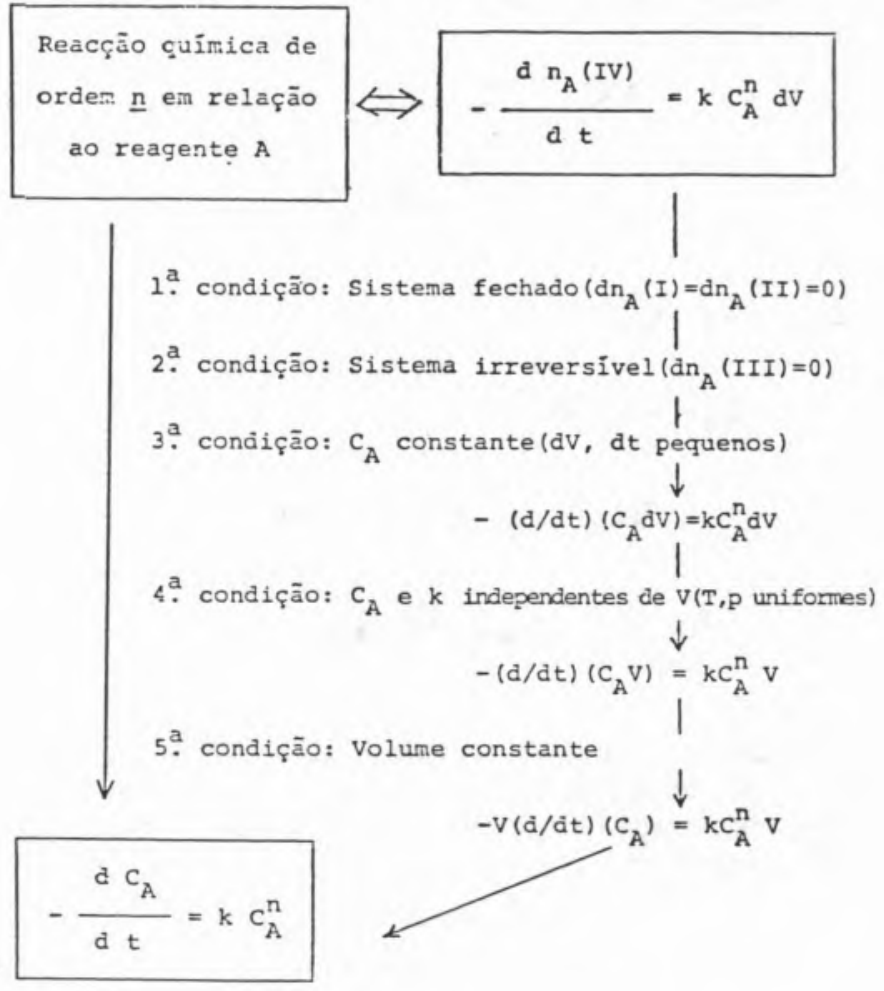

Esquema 1

Lei de velocidade de uma reacção de ordem $n$

a CECUL - Calçada Bento da Rocha Cabral, 14, 1200 Lisboa. 


\subsection{Constantes de velocidades e funções termodi- nâmicas de activação}

A constante de velocidade tem dimensð̃es dependentes da forma da lei de velocidade e do modo como a reacção foi investigada - Tabela 1 .

\begin{tabular}{|ccc|}
\hline $\begin{array}{c}\text { Ordem } \\
\text { de reacção }\end{array}$ & Unidades de k & SI \\
\hline 1 & (tempo $^{-1}$ & $\mathrm{~s}^{-1}$ \\
2 & (concentração) $^{-1}(\text { tempo })^{-1}$ & $\mathrm{dm}^{3} \mathrm{~mol}^{-1} \mathrm{~s}^{-1}$ \\
$\mathrm{n}$ & (concentração) $^{1-\mathrm{n}}(\text { tempo })^{-1}$ & $\mathrm{dm}^{3(\mathrm{n}-1)} \mathrm{mol}^{1-\mathrm{n}} \mathrm{s}^{-1}$ \\
\hline
\end{tabular}

\section{Tabela 1}

Unidades da constante de velocidade

Ao contrário do que acontece para reacçðes de $1 .{ }^{a}$ ordem, as unidades da constante de velocidade de reacçð̌es de ordem superior dependem das unidades em que estão expressas as concentraçðes. Três diferentes escalas de concentração são de uso comum: molar, c, molal, m, e de fracçðes molares, x. A relação entre escalas é simples quando se trata de soluçðes diluidas

$\mathrm{x}=\mathrm{Mc} / 1000 \varrho=\mathrm{Mm} / 1000$

onde $\mathrm{M}$ representa a massa molecular e $\varrho$ a massa especifica, ambas do solvente puro.

A correspondente conversão de constantes de velocidade pode ser feita recorrendo às igualdades:

$\mathrm{k}_{\mathrm{x}}=\mathrm{k}_{\mathrm{c}}(\mathrm{M} / 1000 \varrho)^{1-\mathrm{n}}=\mathrm{k}_{\mathrm{m}}(\mathrm{M} / 1000)^{1-\mathrm{n}}$

A determinação de funçð̃es termodinâmicas de activação a partir da variação da constante de velocidade com as variáveis experimentais temperatura e pressão baseia-se, sempre, em expressðes que incluem $\ln [\mathrm{f}(\mathrm{k})]$; factor este que, por definição, não tem unidades:

$$
\begin{aligned}
& \Delta^{\neq} \mathrm{G}=-\mathrm{RT} \ln \left[\frac{\mathrm{h}}{\mathrm{k}_{8}}\left(\frac{\mathrm{k}}{\mathrm{T}}\right)\right]_{\mathrm{p}} \\
& \Delta^{\neq} \mathrm{H}=\mathrm{RT}^{2}\left[\frac{\partial \ln (\mathrm{k} / \mathrm{T})}{\partial \mathrm{T}}\right]_{\mathrm{p}} \\
& \Delta^{*} \mathrm{~S}=-\left(\frac{\partial \Delta^{\ddagger} \mathrm{G}}{\partial \mathrm{T}}\right)_{\mathrm{p}}=\mathrm{R} \ln \left(\mathrm{h} / \mathrm{k}_{8}\right)+ \\
& +R\left\{\frac{\partial[T \ln (k / T)]_{p}}{\partial T}\right. \\
& \Delta^{\neq} \mathrm{Cp}=\left(\frac{\partial \Delta^{*} \mathrm{H}}{\partial \mathrm{T}}\right)_{\mathrm{p}}=\mathrm{R}\left\{\frac{\partial}{\partial \mathrm{T}}\left[\mathrm{T}^{2} \frac{\partial \ln (\mathrm{k} / \mathrm{T})}{\partial \mathrm{T}}\right]\right\}_{\mathrm{p}} \\
& \Delta^{\ddagger} \mathrm{V}=\left(\frac{\partial \Delta^{\ddagger} \mathrm{G}}{\partial \mathrm{p}}\right)_{\mathrm{T}}=-\mathrm{RT}\left(\frac{\partial \ln \mathrm{k}}{\partial \mathrm{p}}\right)_{\mathrm{T}}
\end{aligned}
$$

Apenas pontualmente os valores das funçð̃es termodinâmicas de activação são independentes das unidades de concentração segundo as quais $\mathrm{k}$ foi definido. São disso exemplo as seguintes igualdades:

$$
\begin{aligned}
\Delta \neq \mathrm{H}_{\mathrm{x}} & =\Delta \neq \mathrm{H}_{\mathrm{m}} \\
\Delta \neq \mathrm{Cp}_{\mathrm{x}} & =\Delta \neq \mathrm{Cp}_{\mathrm{m}} \\
\Delta \neq \mathrm{V}_{\mathrm{x}} & =\Delta \neq \mathrm{V}_{\mathrm{m}}
\end{aligned}
$$

Em geral, no entanto, dependem:

$$
\begin{aligned}
\Delta \neq \mathrm{G}_{\mathrm{x}} & =\Delta \neq \mathrm{G}_{\mathrm{c}}-\mathrm{RT}[(1-\mathrm{n}) \ln (\mathrm{M} / 1000 \varrho)]= \\
& =\Delta \cdot \mathrm{G}_{\mathrm{m}}-\mathrm{RT}[(1-\mathrm{n}) \ln (\mathrm{M} / 1000)]
\end{aligned}
$$

$$
\begin{aligned}
& \Delta^{\ddagger} \mathrm{H}_{\mathrm{x}}=\Delta^{\neq} \mathrm{H}_{\mathrm{c}}+\mathrm{RT}^{2}(1-\mathrm{n}) \varrho \frac{\partial}{\partial \mathrm{T}}\left(\frac{1}{\varrho}\right) \\
& \Delta^{*} \mathrm{~S}_{\mathrm{x}}=\Delta^{*} \mathrm{~S}_{\mathrm{c}}+\mathrm{R}(1-\mathrm{n})\left[\mathrm{T} \varrho \frac{\partial}{\partial \mathrm{T}}\left(\frac{1}{\varrho}\right)+\right. \\
& +\ln (\mathrm{M} / 1000 \varrho)]= \\
& =\Delta^{\ddagger} S_{m}+R(1-n) \ln (M / 1000) \\
& \Delta^{*} \mathrm{C} \mathrm{p}_{\mathrm{x}}=\Delta^{*} \mathrm{C} \mathrm{p}_{\mathrm{c}}+\mathrm{RT}(1-\mathrm{n})\left\{2 \varrho \frac{\partial}{\partial \mathrm{T}}\left(\frac{1}{\varrho}\right)+\right. \\
& \left.+\mathrm{T}\left[\varrho \frac{\partial^{2}}{\partial \mathrm{T}^{2}}\left(\frac{1}{\varrho}\right)+\frac{\partial \varrho}{\partial \mathrm{T}} \cdot \frac{\partial}{\partial \mathrm{T}}\left(\frac{1}{\varrho}\right)\right]\right\} \\
& \Delta^{\neq} V_{x}=\Delta^{\neq} V_{c}-R T(1-n) \varrho \frac{\partial}{\partial \varrho}\left(\frac{1}{\varrho}\right)
\end{aligned}
$$

As equaçðes [12] a [19] foram deduzidas directamente das expressðes [6] a [11].

Embora os valores das funçð̃es termodinâmicas de activação se apresentem, quase sempre, numericamente diferentes consoante as unidades de concentração utilizadas, como vimos, expressam-se nas mesmas unicades: $\mathrm{Jmol}^{-1}$ para a energia de Gibbs e entalpia, $\mathrm{Jmol}^{-1} \mathrm{~K}^{-1}$ para a entropia e capacidade calorífica $\mathrm{e} \mathrm{m}^{3} \mathrm{~mol}^{-1}$ para o volume, no Sistema Internacional.

Por outro lado, as constantes de velocidade de reacçð̃es de ordem $\mathrm{n}$ em fase gasosa expressam-se, por vezes, em unidades de pressão:

$$
\mathrm{k}_{\mathrm{p}} /\left(\text { pressão) }{ }^{1-\mathrm{n}} \text { (tempo) }{ }^{-1}\right.
$$

$\mathrm{O}$ cálculo do parâmetro de Arrhenius, $\mathrm{E}_{\mathrm{a}}$, pode ser feito por duas vias que se indicam no esquema 2 .

Como se vê neste esquema, a utilização de $k_{p}$ no gráfico de Arrhenius conduz a números de colisão diferen-

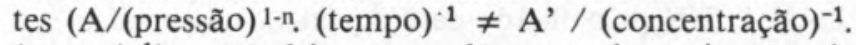
(tempo $^{-1}$ ) e também a parâmetros de activação de Arrhenius, $\mathrm{E}_{\mathrm{a}}\left(\mathrm{k}_{\mathrm{p}}\right)$ e $\mathrm{E}_{\mathrm{a}}\left(\mathrm{k}_{\mathrm{c}}\right)$ numericamente diferentes, embora expressas nas mesmas unidades.

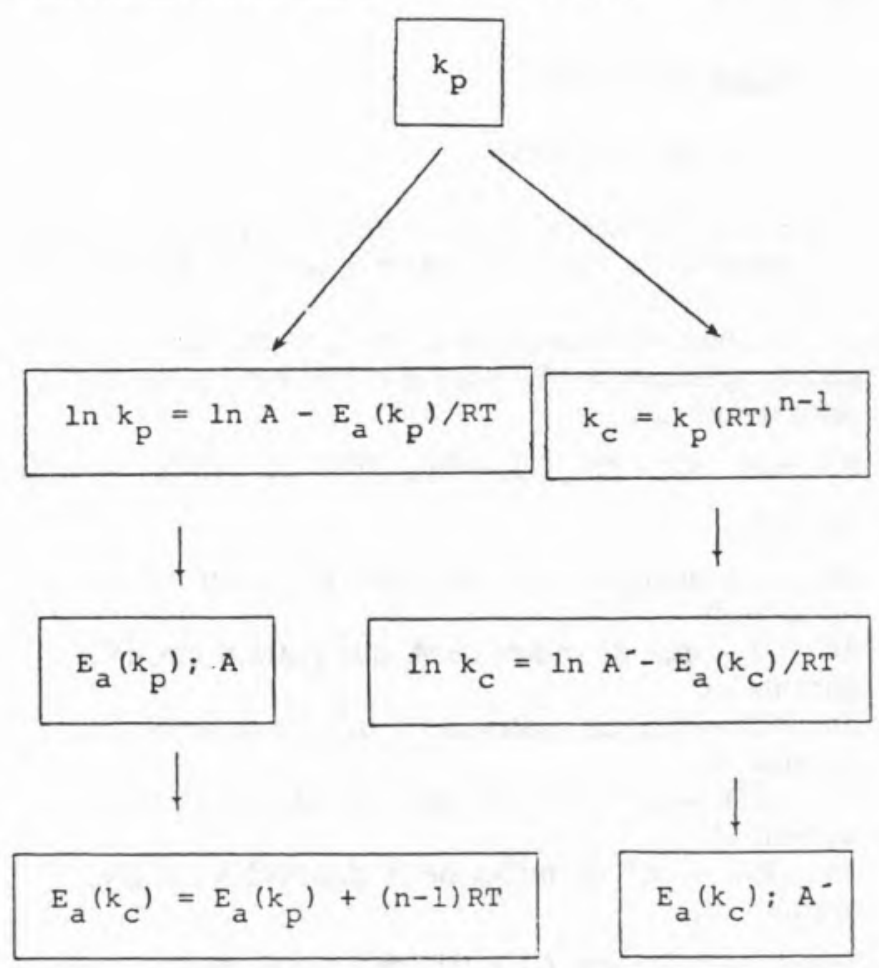




\subsection{Passo determinante da velocidade de reacção}

O passo determinante da velocidade da reacção é habitualmente definido como sendo "o mais lento". Este critério, no entanto, só é útil em sequências de etapas irreversíveis e, mesmo aí, convém explicitar o significado de "o mais lento». No caso simples de duas reacçð̃es irreversíveis consecutivas de $1 .^{\mathrm{a}}$ ordem:

$$
\mathrm{A} \stackrel{\mathrm{k}_{1}}{\longrightarrow}>\mathrm{B} \stackrel{\mathrm{k}_{2}}{\longrightarrow}>\mathrm{C}
$$

uma formulação puramente algébrica, fazendo uso da hipótese de que ao tempo zero, a concentração do reagente $\mathrm{A}$ é $\left[\mathrm{A}_{\mathrm{o}}\right]$ e as de $\mathrm{B}$ e $\mathrm{C}$ são nulas, conduz a:

$$
\begin{aligned}
& {[A]=\left[A_{0}\right] e^{-k_{1} t}} \\
& {[B]=\left[A_{0}\right] k_{1}\left(e^{--k_{1} t}-e^{-k_{2} t}\right) /\left(k_{2}-k_{1}\right)} \\
& {[C]=\left[A_{0}\right]\left[1+\left(k_{2} e^{\left.\left.-k_{1} t-k_{1} e^{-k_{2} t}\right) /\left(k_{2}-k_{1}\right)\right]}\right.\right.}
\end{aligned}
$$

o que se traduziu graficamente na figura 1 .

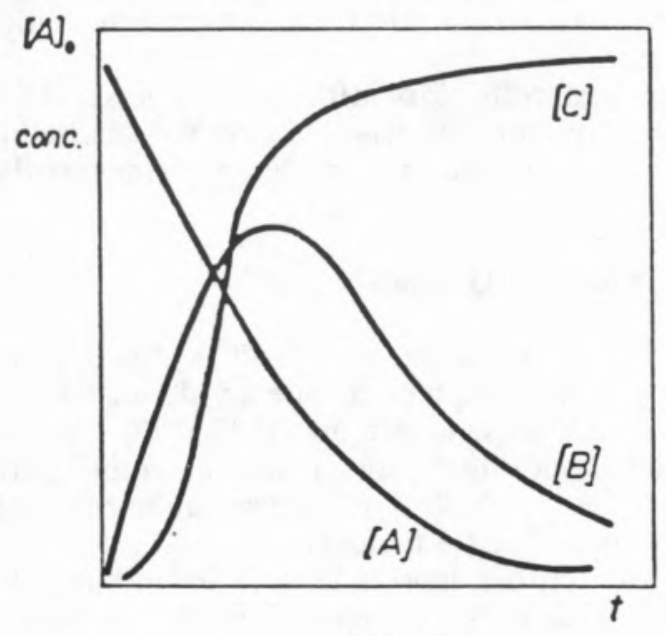

Figura 1

Variação da concentração do reagente $A$, intermediário $B$ e produto $C$ com o tempo

No caso particular de $k_{1} \ll k_{2}$, as equaçðes anteriores reduzem-se a:

$$
\begin{aligned}
& {[A]=\left[A_{0}\right] e^{-k_{1} t}} \\
& {[B]=\left[A_{0}\right] k_{1} e^{-k_{1} t} / k_{2}=[A] k_{1} / k_{2}} \\
& {[C]=\left[A_{0}\right]\left(1-e^{-k_{1} t}\right.}
\end{aligned}
$$

Por uma via independente, aplicando o Princípio do Estado Estacionário ao intermediário B,

$$
\mathrm{d}[\mathrm{B}] / \mathrm{dt}=\mathrm{k}_{1}[\mathrm{~A}]-\mathrm{k}_{2}[\mathrm{~B}]=0
$$

isto é, considerando a velocidade do primeiro passo reaccional, $v_{1}=k_{1}[A]$, igual à do segundo, $v_{2}=k_{2}[B]$, obtem-se o mesmo conjunto de leis cinéticas (equaçðes [23] a [25]).

Nenhuma contradição está implicita no formalismo simultâneo de $k_{1} \ll k_{2}$ e $v_{1}=v_{2}$ desde que se considere $[\mathrm{B}] \ll[\mathrm{A}]$ durante todo o decurso da reacção. A figura 2 exemplifica este caso, entre outros.

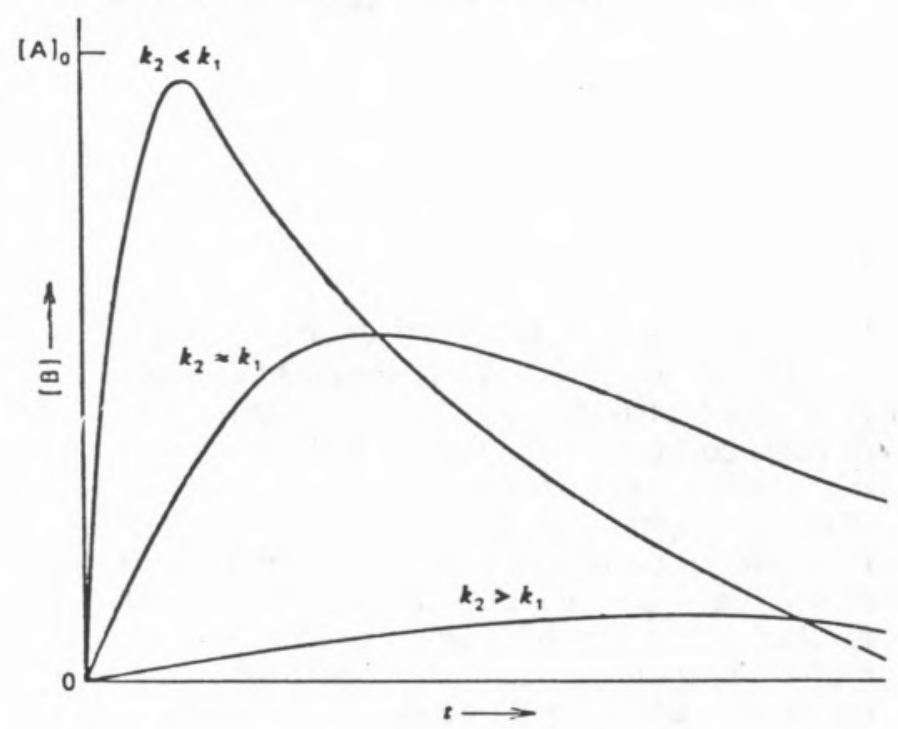

Figura 2

Variação da concentração do intermediário $B$ com o tempo

Consequentemente, o conceito de passo determinante da velocidade reaccional numa sequência de etapas irreversíveis deve ser feito em termos de constantes de velocidade e não de velocidades: o passo "mais lento" é o que apresenta menor valor de constante de velocidade.

Em sistemas mais complexos, reaç̧ðes reversiveis consecutivas, paralelas e competitivas, não é possível uma definição simples de passo determinante de velocidade de reaç̧ão e apenas um exame pormenorizado da estrutura algébrica das equaçðes cinéticas poderá, eventualmente, conduzir à sua identificação.

\section{REFERÊNCIAS}

(1) R.K. Boyd, J. Chem. Educ., 55, 84 (1978).

(2) K. Denbigh, "The Principles of Chemical Equilibrium», Cambridge University Press, $3 .^{a}$ ed., (1971).

(3) P. Zuman e R. Patel, "Techniques in Organic Reaction Kinetics", John Wiley, New York (1984).

(4) M.H. Abraham, Prog. Phys. Org. Chem., 11, 1 (1974).

(5) H. Maskill, Educ. Chem., 22, 154 (1985). 